\title{
Physics inspired by living matter: dynamics, topology and functionality
}

\author{
Arne T. Skjeltorp ${ }^{1,2}$ and Geir Helgesen ${ }^{1,2, a}$ \\ ${ }^{1}$ Institute for Energy Technology, Kjeller, Norway \\ ${ }^{2}$ Department of Physics, University of Oslo, Oslo, Norway
}

Received 18 December 2018

Published online 28 March 2019

The theme of this Special Topics issue, "Physics Inspired by Living Matter", refers in essence to physical phenomena and their description which have been inspired by analogous phenomena occurring in nature. The three subthemes, "Dynamics, Topology and Functionality", are representing the diversity of the contributions linking the dynamics of structural changes to the functionality, i.e. the purpose for which Nature has generated a given structure.

These elements are thus in the forefront of current research in the area of mimicking natural phenomena and biological systems using the tools in physics.

The subthemes are outlined more specifically below.

- Collective dynamics occurs for example in birds flocking, swimming bacteria colonies, and cells forming dynamic aggregates. In these systems, known as active matter, the energy is injected at the level of the "particles". In driven matter the energy is injected at a macroscopic global scale, such as systems subject to vibrations, thermal gradients, electric or magnetic fields. Active matter and driven matter generally exhibit self-assembled or self-organized dynamic structures. Examples of these phenomena are given in the papers by Rasmussen et al. [1], Doostmohammadi and Yeomans [2], Horowitz et al. [3] and Helgesen [4] in this issue.

- Topological features affect the physical properties of matter from nano- to macroscales. Important examples of topological effects are disclinations in liquid crystals, knots and entanglement, twisting and chirality of filaments. Living matter provide numerous examples of such phenomena. Examples of these features are given in the papers by Cabeça et al. [5], Musevic [6] as well as Dietler [7] in this issue.

- Functionality relates to the properties of engineered materials inspired by living matter, such as materials formed by colloidal and polymeric building blocks that can be tuned precisely to achieve macroscopic features such as non-wettability, thermal insulation, or structural coloration. Examples of these properties are given in the papers by Budek et al. [8] as well as Chan and Carlson [9] in this issue.

The papers in this Special Topics issue thus reflect well the diversity of work in this area.

a e-mail: geir.helgesen@ife.no 
This special issue on "Physics Inspired by Living Matter" originated at a recent "Geilo School" (GS), ${ }^{1}$ the twenty-fourth GS in a series held every two years since $1971 .^{2}$

The editors are grateful to Paul Dommersnes, Jon Otto Fossum, and Kenneth Knudsen who helped them choose the themes and selecting the authors to this Special Topics issue.

\section{References}

1. M. Rasmussen et al., Eur. Phys. J. Special Topics 227, 2393 (2019)

2. A. Doostmohammadi, J. Yeomans, Eur. Phys. J. Special Topics 227, 2401 (2019)

3. V.R. Horowitz et al., Eur. Phys. J. Special Topics 227, 2413 (2019)

4. G. Helgesen, Eur. Phys. J. Special Topics 227, 2425 (2019)

5. R. Cabeça et al., Eur. Phys. J. Special Topics 227, 2439 (2019)

6. I. Musevic, Eur. Phys. J. Special Topics 227, 2455 (2019)

7. G. Dietler, Eur. Phys. J. Special Topics 227, 2487 (2019)

8. A. Budek et al., Eur. Phys. J. Special Topics 227, 2495 (2019)

9. T.S. Chan, A. Carlson, Eur. Phys. J. Special Topics 227, 2501 (2019)

\footnotetext{
${ }^{1}$ The Geilo School 2017, 20-30 March, Geilo, Norway. A comprehensive list of lecturers, poster sessions and participants can be found in Physics Inspired by Living Matter: Dynamics, Topology and Functionality (2017) IFE Report IFE/E-2017/002, ISBN (electronic): 978-82-7017-906-0, ISBN (printed): 978-82-7017-905-3.

${ }^{2}$ A list of previous Geilo schools may be found here: http://www.ife.no/departments/physics/ projects/geilo.
} 